\title{
College ERP Using MERN Stack
}

\section{Shubham Patil, Saurav Daware, Ameya Bhagat, Prof. Jayant Sawarkar}

Department of Computer Engineering, Datta Meghe College of Engineering, Airoli, Navi Mumbai, Maharashtra, India

\section{ABSTRACT}

Article Info

Volume 7, Issue 3

Page Number : 190-196

\section{Publication Issue :}

May-June-2021

\section{Article History}

Accepted : 10 May 2021

Published : 16 May 2021
This paper is aimed at developing an Online College Management System that is of importance to the educational institute or college. This system is named College ERP using MERN stack. This system may be used to monitor college students and their various activities. This application is being developed for an engineering college to maintain and facilitate ease of access to information. For this the users must be registered with the system. College ERP is an Internet based application that aims at providing information to all levels of management within an organization. This system is used as an information management system for the college. For a given student and staff (technical and nontechnical) can access the system to either upload and access some information from the database.

Keywords : MERN, ERP, MongoDB

\section{INTRODUCTION}

The title of the project is "College ERP using MERN stack". College ERP is defined as an application based on ternet that aims at all levels of management providing information within an organization. This system can be used as an information management system for the college.

For a given student/staff (Technical / Non-technical) theAdministrator creates a login id \& password, using these students/ staff (Technical / Non-technical) can access the system to either upload or download some information from the database.

The front end will be a React Based javascript application whereas the server will run on Node.js using Express.js. The database side of things will be based on MongoDB whereas Mongoose will help Node.js communicate with the database.

\section{PROBLEM DEFINITION}

The existing system which we are using in our college is a traditional process and is a complete manual process. Now-a-days, education is playing a very significant role in society. Day-by-day, the percentage of illiterates are decreasing and the percentage of literates is increasing. Education will change society in all aspects and everyone wants to study higher professional degrees. Admissions are increasing day by day so the ratio of establishment of new colleges and schools are also increasing. But the actual challenge is starting from now. Most of the schools and colleges maintain student information in records. 
When the number of records increased, it was difficult to maintain the information of each student in the old manual system. Maintaining the records manually leads to error prone and requires more man power and it consumes more time for processing the records.

\section{Aim and Objective}

The main objective of developing the current project entitled "College ERP using MERN stack" is to build an effective system which is fast, accurate, consistent, reliable and flexible enough so that it can incorporate any future enhancements.

By automating the system using computers, sophisticated technology can be used for making the information more flexible, accurate, and secure and user friendly.

Time and manpower can be more effectively utilized and online information can be easily available to the user and at the same time we can maintain a higher level of security.

Every user such as student, staff and administration is able to see the information of each student online from anywhere and anytime.

\section{Literature Review}

Until recently the place of the College Management System (CMS). Now-a-days, education is playing a very significant role in society. Day-by-day, the percentage of illiterates are decreasing and the percentage of literates is increasing. Education will change society in all aspects and everyone wants to study higher professional degrees.

Admissions are increasing day by day so there by. Ratio of establishment of new colleges and schools are also increasing. But the actual challenge is starting from now. Most of the schools and colleges maintain student information in records.

When the number of records increased, it was difficult to maintain the information of each student in the old manual system.

Maintaining the records manually leads to error prone and requires more man power and it consumes more time for processing the records.

\section{Requirement Analysis}

\section{Software Requirements}

We need the following software to accomplish our project.

1. Writing Code (Sublime Text)

2. MERN Stack

a. MongoDB: A cross-platform document database.

b. Express: A back-end web application framework.

c. React: A JavaScript library for building user interfaces.

d. Node.js: A cross-platform JavaScript runtime environment.

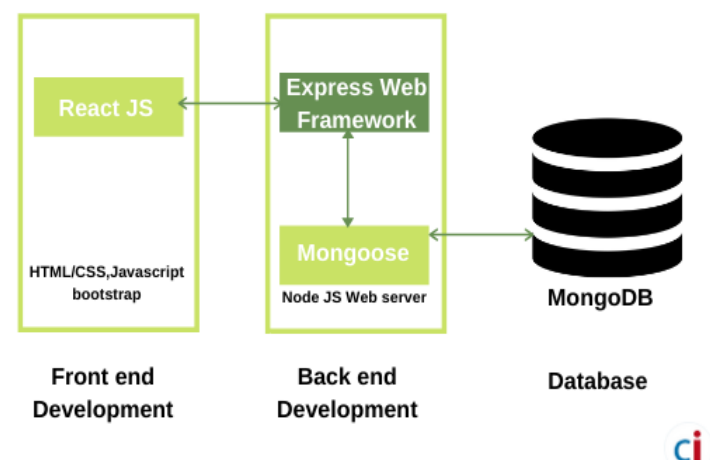

Figure 1. MERN stack

3. Web Browser for accessing the website. 


\section{Hardware Requirements}

We will need the following hardware to accomplish our project.

1. Desktop or Laptop PC with Windows 10 64bit with 8GB Ram and atleast 2GB of free space.

2. Android or iPhone that can run the latest version of web browsers like Chrome, Safari or firefox.

\section{Project Design and Modules}

\section{Login Page}

Login Page will be used to authenticate various users that will be using the system.

College ERP
\#\#Admin $\quad$ Dsaculty

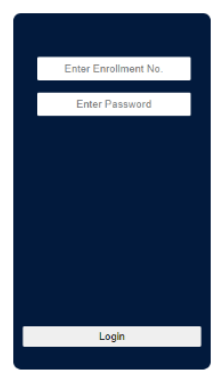

Figure 2. Login Page

\section{Admin Dashboard}

This page will provide admin with the tool to manage all the students and faculties as well as other tools.

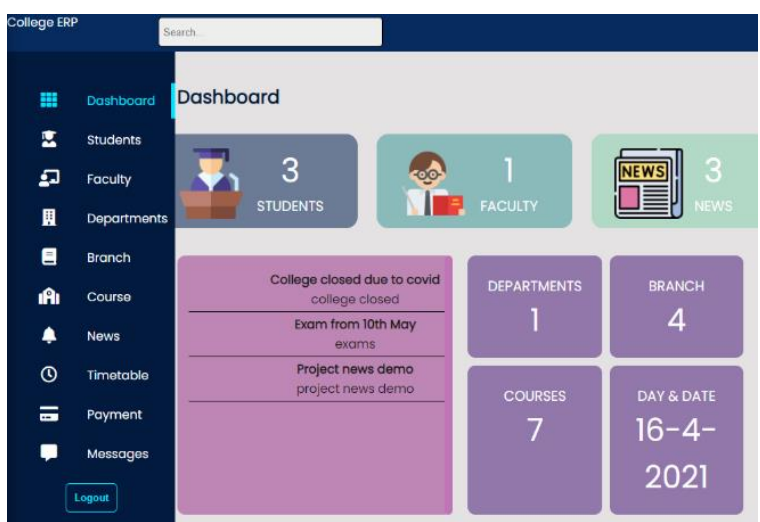

Figure 3. Admin Dashboard

These are the various modules available for the admin.

1. Students

2. Faculty

3. Departments

4. Branch

5. Course

6. News

7. Timetable

8. Payment

9. Messages

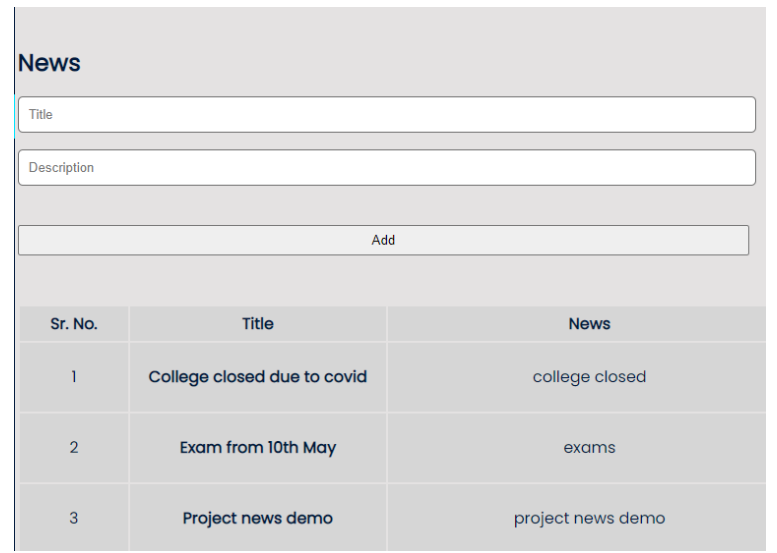

Figure 4. News Page

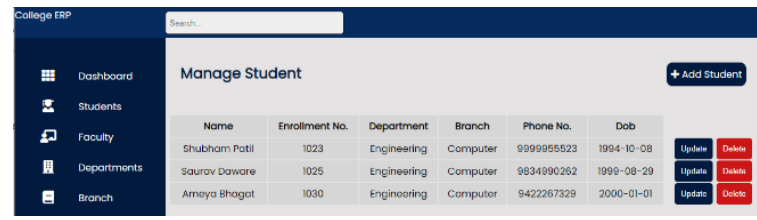

Figure 5. Students Information 
Shubham Patil et al Int. J. Sci. Res. Comput. Sci. Eng. Inf. Technol, May-June - 2021, 7 (3) : 190-196

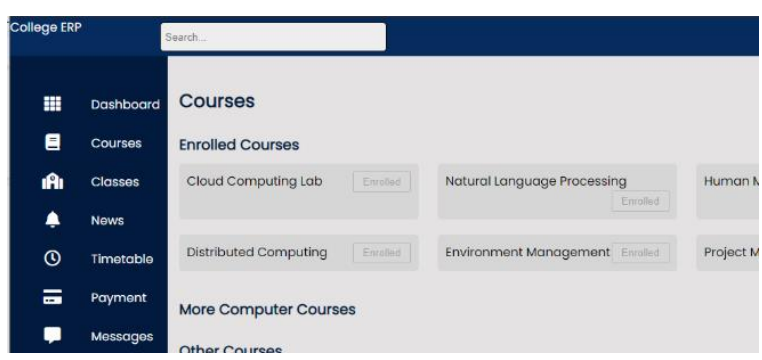

Figure 6. Student Subject Enrollment

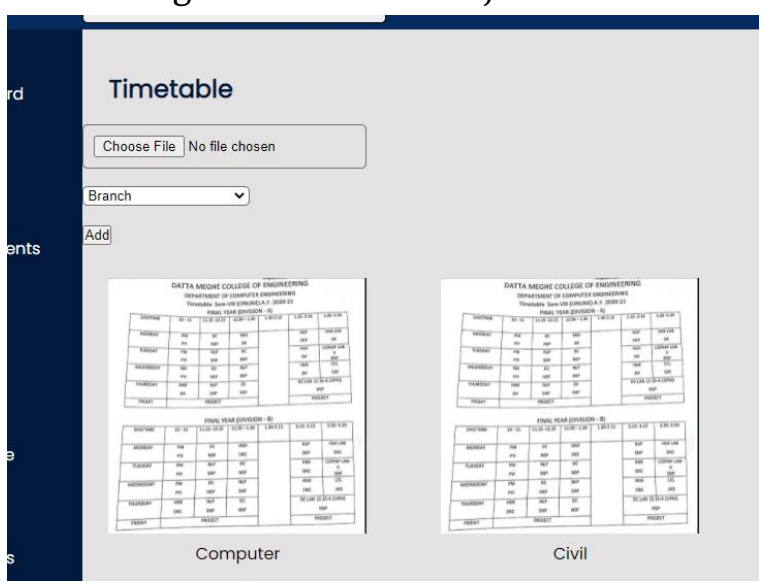

Figure 7. Timetable Management

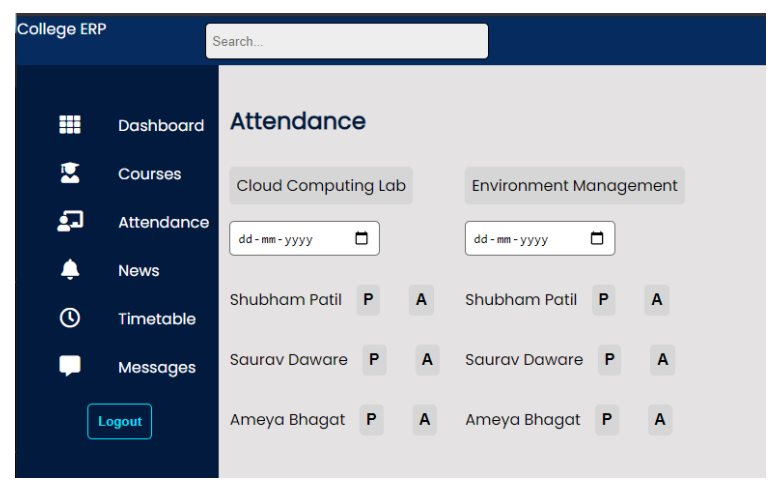

Figure 8. Attendance Page

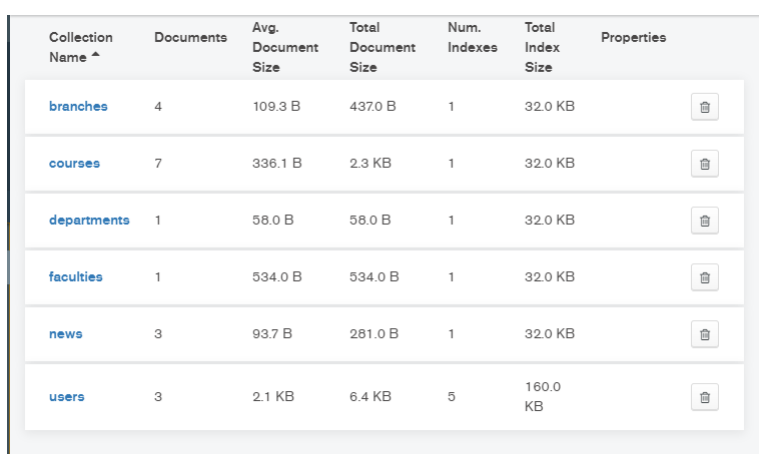

Figure 9. MongoDB Compass

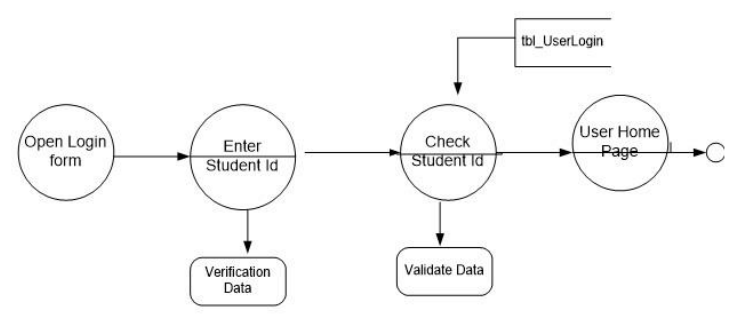

Figure 10. Login DFD

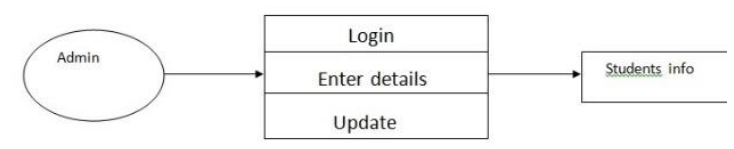

Figure 11. 1st level DFD Admin
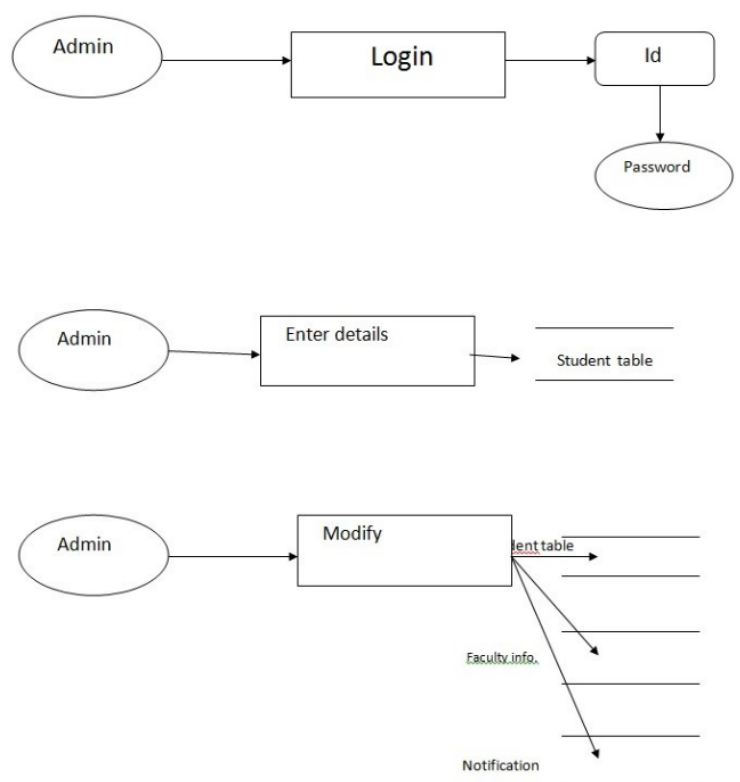

Figure 12. 2nd Level DFD Admin

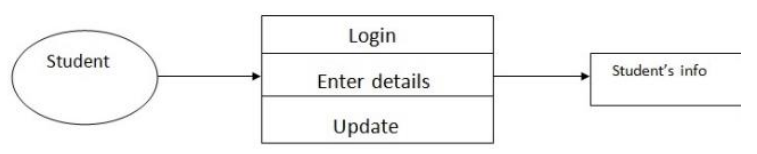

Figure 13. Student data Flow 


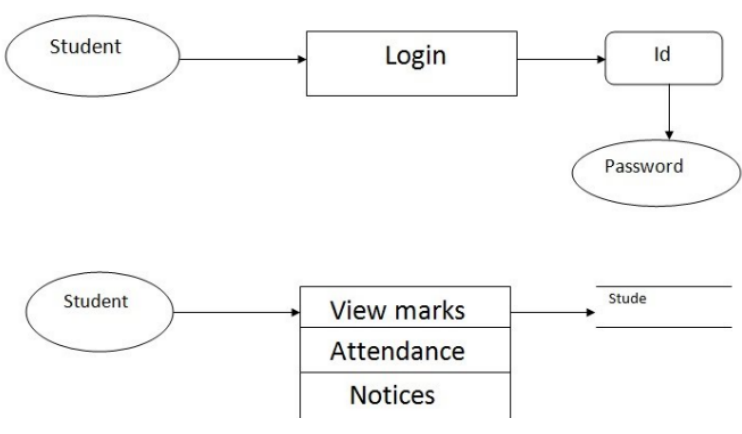

Figure 14. Student 2nd Level DFD

\section{Technologies Used}

HTML: The HyperText Markup Language, or HTML is the standard markup language for documents designed to be displayed in a web browser. It can be assisted by technologies such as Cascading Style Sheets (CSS) and scripting languages such as JavaScript.

CSS: Cascading Style Sheets (CSS) is a style sheet language used for describing the presentation of a document written in a markup language such as HTML. CSS is designed to enable the separation of presentation and content, including layout, colors, and fonts. This separation can improve content accessibility, provide more flexibility and control in the specification of presentation characteristics, enable multiple web pages to share formatting by specifying the relevant CSS in a separate .css file which reduces complexity and repetition in the structural content as well as enabling the .css file to be cached to improve the page load speed between the pages that share the file and its formatting.

Javascript: JavaScript is one of the core technologies of the World Wide Web. Over 97\% of websites use it client-side for web page behavior, often incorporating third-party libraries. All major web browsers have a dedicated JavaScript engine to execute the code on the user's device.
As a multi-paradigm language, JavaScript supports event-driven, functional, and imperative programming styles. It has application programming interfaces (APIs) for working with text, dates, regular expressions, standard data structures, and the Document Object Model (DOM).

MERN stack: The MERN architecture allows you to easily construct a 3-tier architecture (frontend, backend, database) entirely using JavaScript and JSON.

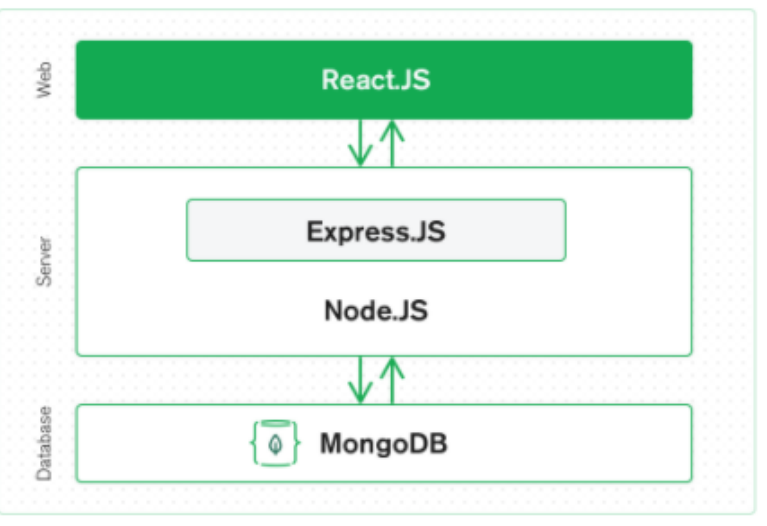

Figure 15. MERN Stack

\section{React.js Front End}

The top tier of the MERN stack is React.js, the declarative JavaScript framework for creating dynamic client-side applications in HTML. React lets you build up complex interfaces through simple Components, connect them to data on your backend server, and render them as HTML.

\section{Express.js and Node.js Server Tier}

The next level down is the Express.js server-side framework, running inside a Node.js server. Express.js bills itself as a "fast, unopinionated, minimalist web framework for Node.js," and that is indeed exactly what it is. Express.js has powerful models for URL routing (matching an incoming URL with a server function), and handling HTTP requests and responses. 


\section{MongoDB Database Tier}

If your application stores any data (user profiles, content, comments, uploads, events, etc.), then you're going to want a database that's just as easy to work with as React, Express, and Node.

That's where MongoDB comes in: JSON documents created in your React.js front end can be sent to the Express.js server, where they can be processed and (assuming they're valid) stored directly in MongoDB for later retrieval. Again, if you're building in the cloud, you'll want to look at Atlas. If you're looking to set up your own MERN stack, read on!

\section{CONCLUSION}

College ERP Management using MERN Stack provides an easy way to automate the functionalities of the college. It is an integrated platform that connects various departments of an institution like administration, staff, students as well as guardians. It provides reliability, time savings, and is easy to control. Information can be saved and can be accessed at any time by an authorized user. It includes almost all modules required for seamless college functions.

These modules include attendance management, railway concession system, training and placement, staff details, staff records, etc. It also facilitates exploring various events happening in the college such as exams, practical, submission, placements, annual events in the form of notifications.

\section{ACKNOWLEDGEMENT}

Motivation and guidance are the keys towards success. I would like to extend my thanks to all the sources of motivation.
We would like to grab this opportunity to thank Dr.

S. D. Sawarkar, principal for encouragement and support he has given for our project.

We express our deep gratitude to Dr. A. P. Pande, head of the department who has been the constant driving force behind the completion of this project.

We wish to express our heartfelt appreciation and deep sense of gratitude to my project guide Prof. J. D. Sawarkar for his encouragement, invaluable support, timely help, lucid suggestions and excellent guidance which helped us to understand and achieve the project goal. his/her concrete directions and critical views have greatly helped us in successful completion of this work.

We extend our sincere appreciation to all professors for their valuable inside and tips during the designing of the project. Their contributions have been valuable in so many ways that we find it difficult to acknowledge them individually.

We are also thankful to all those who helped us directly or indirectly in completion of this work.

\section{REFERENCES}

[1]. https://www.slideshare.net/mohit0749/collegemanagement-system-project-48367405

[2]. https://1000projects.org/college-managementsystem-cms.html

[3]. (PDF) A Research Paper on College Management System | suraj kumar Academia.edu https://www.academia.edu/35401042/A_Resear ch_Paper_on_College_Management_System

[4]. MERN Stack https://www.geeksforgeeks.org/mern-stack/

[5]. https://www.ijarcce.com/upload/2013/june/4shobha\%20bharamaoudar- 
WEB\%20BASED\%20STUDENT\%20INFORMA TION.pdf

[6]. https://en.wikipedia.org/wiki/HTML

[7]. https://en.wikipedia.org/wiki/JavaScript

[8]. https://en.wikipedia.org/wiki/CSS

[9]. https://www.mongodb.com/mern-stack

[10]. https://sites.google.com/site/ignoubcafinalyearp rojects/project-report/college-managementsystem-projectreport?tmpl=\%2Fsystem\%2Fapp\%2Ftemplates \%2Fprint\%2F\&showPrintDialog=1

\section{Cite this article as :}

Shubham Patil, Saurav Daware, Ameya Bhagat, Prof. Jayant Sawarkar, "College ERP Using MERN Stack", International Journal of Scientific Research in Computer Science, Engineering and Information Technology (IJSRCSEIT), ISSN : 2456-3307, Volume 7 Issue 3, pp. 190-196, May-June 2021. Available at doi : https://doi.org/10.32628/CSEIT217337

Journal URL : https://ijsrcseit.com/CSEIT217337 